\title{
Spectra of the photo-electric phenomena physically differentiated on the light absorption factor
}

\author{
L.I. Berezhinsky, E.F. Venger, I.E. Matyash, B.K. Serdega \\ V. Lashkaryov Institute of Semiconductors Physics, NAS of Ukraine \\ 41, prospect Nauky, 03028 Kyiv, Ukraine \\ Phone: 525-57-78 \\ E-mail: serdega@isp.kiev.ua
}

\begin{abstract}
The pleochroism phenomena in photoconductivity of Ge samples and gate photoelectomotive force (photo-emf) of Si samples were experimentally investigated by a polarization modulation method. Anisotropy of dielectric properties was created by uniaxial compression. The spectral characteristics of the polarization difference describing above effects were measured. We found that: a) the pleochroism spectrum of photoconductivity is a derivative with respect to the absorption factor of a photocurrent function; b) the pleochroism spectrum of the gate photo-emf contains dichroic components, determined by the sample thickness, diffusion length of majority carriers, and layer thickness of a spatial charge.
\end{abstract}

Keywords: polarization-modulation spectroscopy, semiconductor, photoconductivity, gate photoelectromotive force.

Manuscript received 08.11.04; accepted for publication 16.12.04.

\section{Introduction}

As it is well known [1], the light absorption factor $\alpha$ of substance is precisely parameter that defines the shape of spectral characteristics of photoelectric effects. So, photoconductivity and various photovoltaic effects present in semiconductor crystals with various values of the forbidden band $E_{g}$, have such a general feature as identical dependences of the effect magnitude on the values of the light absorption factor. Therefore, the relation of the crystal parameters $\alpha$ and $E_{g}$ with each other remains an actual task, which can be solved by various ways. One of them based on the principle of modulation spectroscopy and designated as $\lambda$ modulation is investigated in details and has a wide practical application [2]. However, among the known set of modulation methods, there exist no one capable to modulate any spectral characteristic either optical or photoelectric effect by the light absorption factor. We keep in mind that, during measurements of the effect spectral dependence, the $\alpha$-magnitude varies within narrow limits around the current value. But any physical action on the crystal (electric or magnetic field, deformation) that varies $\alpha$, also modulates simultaneously a number of other crystal parameters width of the forbidden gap, effective mass of carriers, etc.

Earlier we demonstrated [3] with an example of dichroism phenomenon in uniaxial strained silicon crystals that the application of polarized modulation (PM) radiation, results in obtaining the spectral characteristic that was a derivative of the sample transmission function $T(\alpha)$ with the absorption factor multiplied by the anisotropy value $\Delta \alpha: \Delta T=(d T / d \alpha) \Delta \alpha$. This equality is true if the magnitude of anisotropy is small $\left(\alpha_{\perp}-\alpha_{\|}\right)<<\alpha_{\perp}, \alpha_{\|}$, where $\alpha_{\perp}$ and $\alpha_{\|}$are the absorption factors of light for perpendicular and parallel orientations, respectively, of the wave electric vector $\mathbf{E}$ relatively to the crystal optical axis. As regard to the application of PM in studying the photoelectric effects, the problem remains open. It is possible only to assume that, in contrast to the dichroism observed in transmittance and described by Lambert's law, this parameter will undergo essential changes in photoelectric effects (where the term pleochroism is used instead of dichroism). The basis for this assumption is the fact that the stationary distribution of nonequilibrium carriers generated by absorbed radiation in a sample differs from the exponential one due to a contribution of diffusion processes into photoconductivity and photovoltaic effects.

At the same time, it is necessary to take into account that real crystals have internal mechanical tensions, the origin of which is caused by uncontrolled heterogeneities formed during their growth or due to special doping when manufacturing their various structures. The distribution of heterogeneities is generally characterized by a concentration gradient. As a 


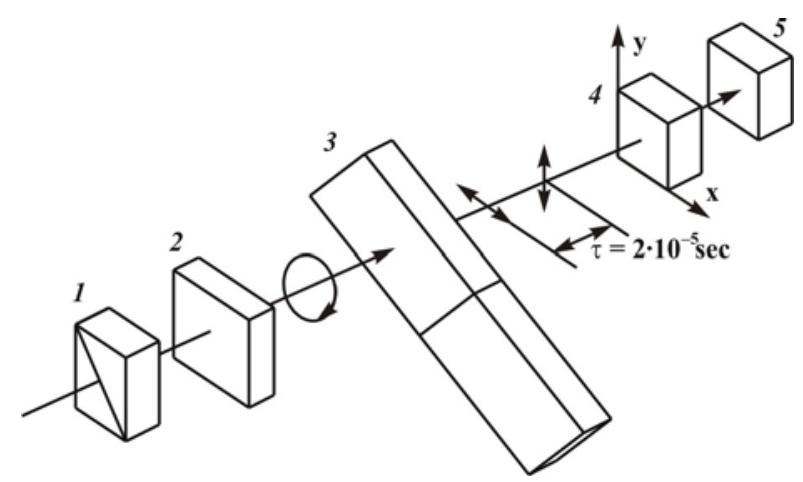

Fig. 1. Optical scheme of setup for measurements of the linear dichroism in an anisotropic crystal:

1 - polarizer, 2 - quarter-wave achromatic phase plate, 3 photoelastic modulator of polarization, 4 - sample, 5 photodiode.

result, there arises some uniaxial deformation and consequently anisotropy of dielectric properties in cubic crystals. The interaction of the polarized radiation with such substances is accompanied by the dichroic effect that is absorption (or transmittance) dependence on an azimuth of the linearly polarized radiation relating to the direction of deformation.

The task of this work is to determine special features connected with PM application in photoelectronics and clarify opportunities of physical differentiation of spectral dependences by the absorption factor of light.

\section{Experimental}

Of a set of photoelectric effects the photoconductivity and gate photoelectromotive force (photo-emf) were studied in this work. It is caused by the fact of a wide use of these phenomena in laboratory practice and in technical applications. The argument in support of a choice of these materials is as follows. Ge crystal is the most convenient for measurements of the photopleochroism magnitude. The large lifetime and wide range of surface recombination speed variations by a simple way make this material suitable for obtaining the set of photoconductivity spectral characteristics. Regarding to the photovoltaic effect, we used photo-emf of the $p-n$ junction as an element that transforms electromagnetic radiation into an electrical signal. However, in the case of a dichroism research, it is not only as researched sample, but acts as a detector of transmitted radiation. It is also applicable to the photocurrent function for the research of dichroism in photoconductivity.

Samples used in this work are plane-parallel plates cut out in such a manner that (100) crystallographic plane of a crystal coincided with an illuminated surface. By means of ohmic contacts, the samples were connected to external circuit consisting of a constant voltage source and loading resistance, the magnitude of which is much less than resistance of samples. So, the gate photo-emf as well as the photoconductivity was measured in a photocurrent mode.

The samples for measurements of photoconductivity prepared from the poorly doped $\mathrm{Ge}$ crystal ( $\rho=5 \mathrm{Ohm} \cdot \mathrm{cm}$ at $300 \mathrm{~K}$ ) of $n$-type so that, after cutting and standard treatment, it had a plate shape with the dimensions $1 \times 0.5 \times 0.2 \mathrm{~cm}^{3}$. Ohmic contacts were placed at the narrow end faces, and the wide surface coincident with (100) crystallographic plane was illuminated by the polarized radiation incident along the normal to the surface. The various treatments of an illuminated surface resulted in different values of the surface recombination speed: etching in hydrogen peroxide $-50 \mathrm{~cm} / \mathrm{s}$, wiping off by a dry paper $-10^{3} \mathrm{~cm} / \mathrm{s}$, mechanical polishing $10^{4} \mathrm{~cm} / \mathrm{s}$.

For the measurement of the gate photo-emf, the samples of measures $1 \times 0.5 \times 0.04 \mathrm{~cm}^{3}$ were broken off parallel to $\{110\}$ planes of standard silicon $\left(\rho=5 \cdot 10^{3} \mathrm{Ohm} \cdot \mathrm{cm}\right.$ at $300 \mathrm{~K}$ ) plate of $n$-type and $0.4-\mathrm{mm}$ thickness. The normal to this plate had the direction [100]. $p$-type conductivity was provided by implantation on one of the surfaces. The sample was connected to the testing circuit by aluminium electrodes (continuous on the back surface and as a ring on the illuminated one). A compressing uniaxial effort was applied to the narrow end faces of Ge or Si plate by such way that its direction was parallel to the illuminated surface and coincided with [110] crystallographic direction. The sample was located in the compressing device, where the special fastening provided a condition of its steady state. It is of the first importance during the compression of thin samples to obtain a homogeneous distribution of the mechanical pressure.

Here it is pertinent to mention that, in this case, the photopleochroism is caused by structural anisotropy. The latter was generated by uniaxial strain of cubic syngony crystals which were used in this work. Therefore, light propagation along $z$ axis, being a normal to an illuminated surface, is accompanied by a difference in absorption of ordinary and extraordinary beams. Thus, our investigation method differs from those in publications such as [4], where the nature of photopleochroism called as "induced", correlated with Fresnel conditions that describe special features of reflection and absorption at oblique incidence of linear polarized light onto a crystal surface.

The measurements were carried out using the setup with the optical scheme depicted in Fig. 1. Here, as a source of constant intensity monochromatic light in the proper energy range used was the diffractional monochromator MDR-12 with a halogen lamp KGM150 at the input. After passing the monochromator, the light propagated through a polarizer 1, achromatic quarter-wave phase plate 2 and a result has taken a circular polarization. Further it was directed on the modulator of polarization 3, researched sample 4, and photodiode 5 . 

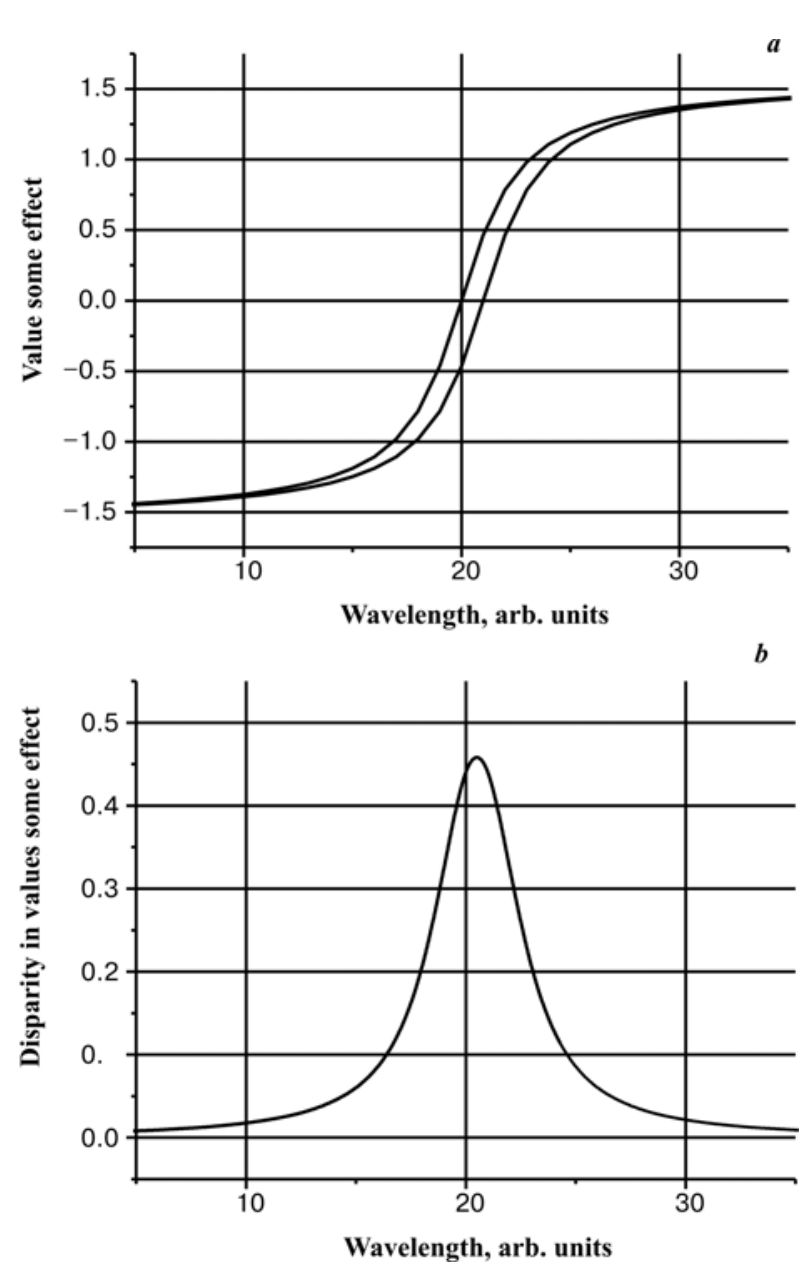

Fig. 2. Schematic spectral characteristics of the optical or photoelectric effects in anisotropic substance measured for two states of light polarization (a), in comparison with the characteristic of polarization difference obtained by the PM method (b).
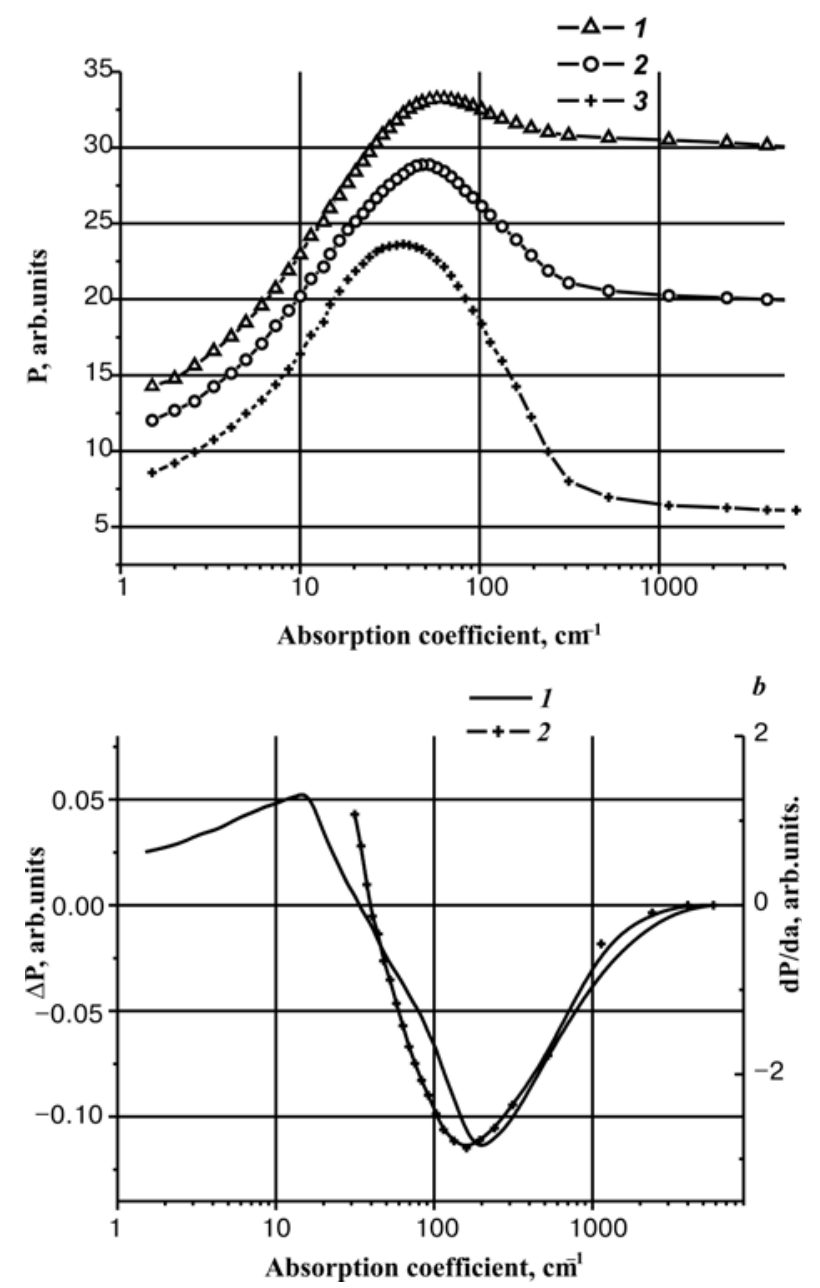

Fig. 3. Spectral characteristics:

a) the photoconductivity of Ge sample loaded by the uniaxial compression $3 \mathrm{kbar}$ at various values of the surface recombination rate: $1-10^{2}, 2-10^{3}, 3-$ $10^{4} \mathrm{~cm} / \mathrm{s}$

b) the polarization difference of photoconductivity of the same sample (curve 1) and derivative of photoconductivity (curve 2 ) at the value of the surface recombination rate $10^{4} \mathrm{~cm} / \mathrm{s}$.

optical axis, it is possible to achieve its arrangement relatively to the sample in such a manner that the planes of light polarization are parallel to $x$ and $y$ axes of the sample (Fig. 1).

The various absorption factors of light in the anisotropic sample cause changes in the intensity of transmitted light that was registered by the photodiode 5 . In the case of photoconductivity, the intensity of absorption light is determined by the photocurrent value (with account of carriers diffusion), and by the potential difference on $p$ - $n$ junction for the gate photo-emf. The photodiode signal was registered by the usual amplitude modulation method, and after synchronous detection it passed to analog graph plotter. 


\section{Results and discussion}

Before discussing the obtained results, we shall consider the process providing the polarization difference of two functions. As it is well known, in the case of uniaxial strain of cubic crystals, the selection rules for interband transitions become dependent on an azimuth of light linear polarization owing to removal of valency band degeneration at the centre of the Brillouin zone. As a result, there is a bifurcation of the edge of the spectral dependence for the absorption factor, and consequently of photoelectric effect spectra, as is schematically shown in Fig. 2a (curves 1 and 2). Since the widths of the forbidden gaps for orthogonal polarizations become different, there is a shift along the energy scale for one of the spectral dependences of the effect (and consequently along the scale of absorption factors). If the difference between peak values of dependences is comparable with their values (this situation takes place in strongly anisotropic crystals in the large curvature region for studied characteristics), the measurement of pleochroism using the traditional way presents no problems. If the value of pleochroism is comparable with values of errors (noise, nonrepeatability) only the polarization modulation should be used. Schematically, this process is shown in Fig. 2, where the curve 2a represents the transmittance of light by hypothetical substance, and the curve $2 \mathrm{~b}$ is the practically momentary difference between curves 1 and 2 (Fig. 2a). The shape of this characteristic looks like the curve of single sign in the absence of a peak in the spectral characteristics, as shown in the scheme (Fig. 2a). It is not difficult to imagine that, in the presence of a peak in the curves of Fig. 2a, they will intersect at some point. In this case, a polarization difference will be registered as alternating curve.

Fig. 3 shows the results of measurements of photoconductivity $P(\alpha)$ in Ge sample. Here, the values of photoconductivity as a function of the absorption factor are represented for various values of the surface recombination speed. Experimentally, the photoconductivity was measured as a function of the wavelength. Then the argument was recalculated from the wavelength to the values of the absorption factor in view of the data [5], correlating the absorption factor to the quantum energy. The interpretation of dependences in Fig. 3a does not involve difficulties because their shape corresponds to usual representations of bipolar photoconductivity with respect to the relation between the rates of volume and surface recombinations. From Fig. 2b, it is clear why the spectral characteristics of the photoconductivity polarization difference $\Delta P$ have the shape of an alternative curve. In Fig. $3 b$, the curve 1 shows $\Delta P$ for the case $s=10^{4} \mathrm{~cm} / \mathrm{s}$. In the same figure, for comparison (curve 2), shown is the derivative $d P / d \alpha$ of isotropic photoconductivity with respect to the absorption factor obtained by graphic differentiation of the curve 3 (Fig. 3a). The satisfactory coincidence of
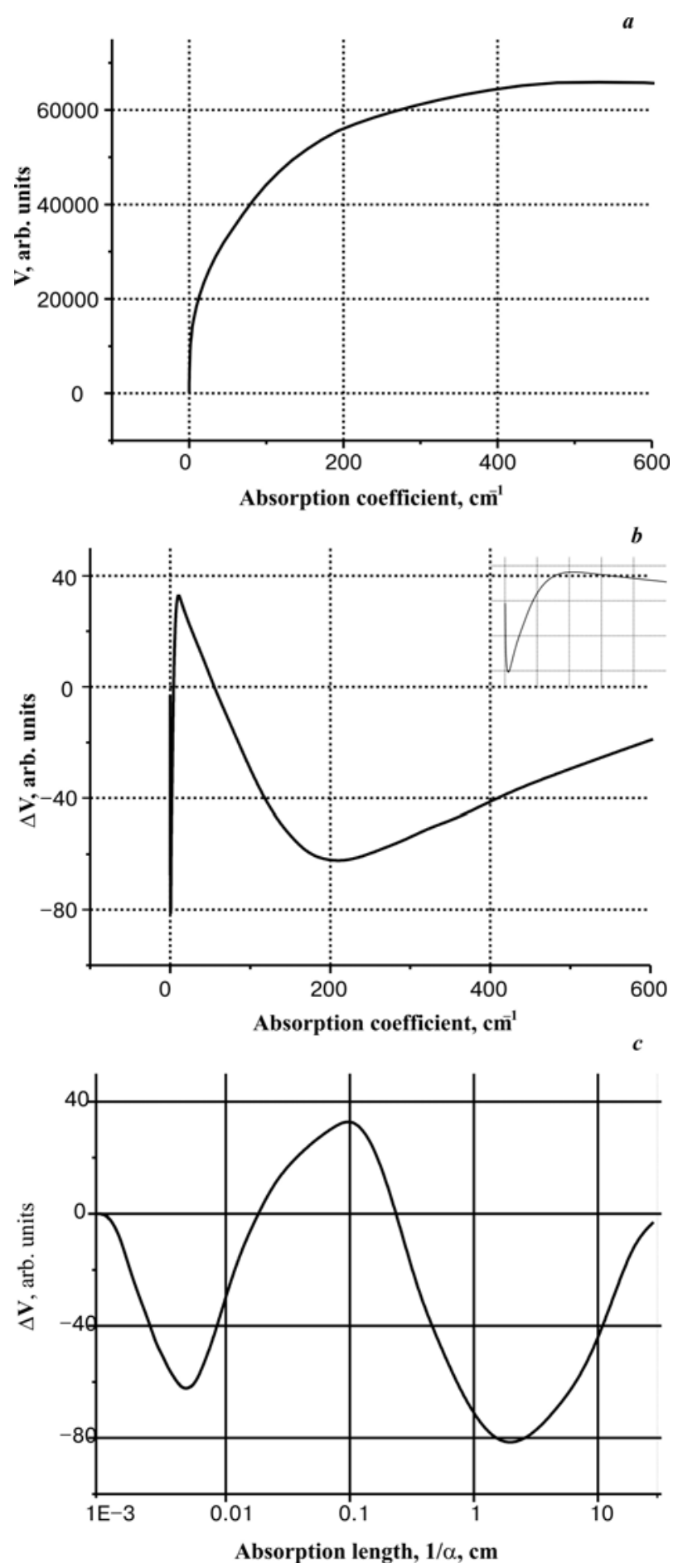

Fig. 4. Spectral characteristics:

a) the gate photo-emf $V$ of $\mathrm{Si} p$ - $n$ junction loaded by uniaxial compression $1 \mathrm{\kappa bar}$,

b) the polarization difference in the range of small absorption factors; in insert, it is shown a polarization difference $\Delta V$ of the gate photo-emf;

c) the dependence of the value of the polarization difference $\Delta V$ of the gate photo-emf (the curve in Fig. $4 \mathrm{~b})$ on the reverse absorption factor of light. 
these curves in the range of complete light absorption within the sample thickness limits corresponds to the conception of $\alpha$-modulation, similarly to the $\lambda$ modulation described in [3]. Moreover, it was determined experimentally that the occurrence of a negative extremum in the curve 1 is caused by increasing the value $s$. This fact testifies about the existence of "dichroism" in the part of the sample, which is determined by the diffusion length of charge carriers.

The spectral characteristics of gate photo-emf $V$ for Si sample and its polarization difference $\Delta V$ are depicted in Fig. 4. The absence of an extremum and recession in the dependence $\Delta V(\lambda)$ (Fig. 4a) in the area of increased absorption factor testifies the absence or at least a negligible recombination rate in the $p-n$ junction area. Regarding to the spectrum of a polarization difference of a gate photo-emf (Fig. 4b), it is seen even without the detailed analysis that, in this case, the curve shape mismatches to that of the derivative of the isotropic characteristic. Nevertheless, it is possible to draw some conclusions when being based on this curve, if to reconstruct it to the argument of $1 / \alpha$ (Fig. $4 \mathrm{c}$ ). First of all, equality to zero of the difference $\Delta V$ when $1 / \alpha \sim 10^{-3} \mathrm{~cm}$ testifies that both exponential absorption functions, corresponding to longitudinal and transversal polarization, are along this length. However, due to the high specific resistance, the area of a spatial charge $w$ in the base of $p$ - $n$ junction becomes so large, that it allows a dichroism to be shown on the length of absorption, which is comparable with the magnitude of $w$, corresponding to formation of an extremum at $1 / \alpha \approx 5 \cdot 10^{-3} \mathrm{~cm}$. The next zero value of $\Delta V$ is observed when $1 / \alpha \approx 1.7 \cdot 10^{-2} \mathrm{~cm}$. It means that, already on the diffusion length, there is no distinction in absorption of both polarizations, i.e. both of those are absorbed completely. So, if the sample thickness $d$ is much larger than the diffusion length, the next extremum of $\Delta V$ may appear for the argument values on the abscissa, which are comparable with the value of the diffusion length. In reality, extremum of $\Delta V$ appears at the argument much larger than the diffusion length, because, in this case, the sample becomes transparent, and role of the component of a polarization difference caused by reflection from metallized back surface of a sample is significant. Under the condition of weak absorption $(\alpha<<1 / d)$, this component is the cause of dichroism on a sample thickness.

\section{Conclusion}

Our researches allowed us to draw a conclusion that the process of physical differentiation of spectral dependences of photoelectric effects with regard to the absorption factor according to the condition $\Delta F=(d F / d \alpha) \Delta \alpha$, where $F$ is the peak value of the effect, does not take place in any case. It was earlier determined that this condition is well be met for the transmittance effect [3]. In this work, it is shown that, in the case of photoconductivity (Fig. 3b), the condition is met in a lesser degree, and, in the case of the gate photo-emf (Fig. 4b), it is met still less. And it is clear, in a transmittance case, the cause of dichroism is the difference in distributions of light intensities along its propagation in a crystal described by the Lambert law. In a case of photoelectric effects, the distribution of charge carriers generated by light (exponential according to the Lambert law) are deformed by diffusion (for photoconductivity) or diffusion-drift (for $p-n$ junction) processes. Therefore, the interpretation of the spectra of the polarization difference demonstrating a certain informative ability of photoelectric effects can become a basis of diagnostics of materials and devices. So, using PM in the weekly strained crystals, it is possible to rather simply estimate the diffusion length of charge carriers or size of a spatial charge area in photodiodes from the analysis of the spectral characteristics of a pleochroism.

It is necessary to add, that the opportunities of the PM method are characterized by the detectability of devices, which is used in the PM method. So, in this work, using the example of measuring the linear dichroism value (photopleochroism) as an illustration of reliability of the obtained results, it is possible to consider the ratio $\Delta V / V$. The value of this ratio taken from ordinates of Figs $4 \mathrm{a}$ and $\mathrm{b}$ does not exceed the value 0.001 ; at the same time, the absolute $\Delta V$ value is an order of magnitude higher, on the average, than the level of the measured system noise.

\section{References}

1. T. Moss, Photoconductivity // Repts Progr. Phys. 28, p. 15-60 (1965).

2. M. Cardona, Modulation spectroscopy, Mir, Moscow, 1972.

3. S.V. Kondratenko, I.E. Matyash, B.K. Serdega // Semiconductor Physics, Quantum Electronics and Optoelectronics 7 (2), p. 195 (2004).

4. F.P. Kesamanly, V.Yu. Rud', Yu.V. Rud'// Fiz. Tekhn. Poluprov. 33 (5), p. 513 (1999).

5. W.C. Dash and F. Newman // Phys. Rev. 99, p. 1151 (1955). 